\title{
Accidental endoscopic finding of Anisakis simplex in human colon
}

\author{
Aurelia Aloia', Pasquale Carlomagno², Michele Gambardella², Manlio Schiavo', \\ Vincenzo Pasquale ${ }^{4}$ \\ I Laboratorio Schiavo, 84078 Vallo della Lucania, Salerno, Italy \\ 2 Clinica Cobellis, 84078 Vallo della Lucania, Salerno, Italy \\ 3 Ospedale S. Luca, 84078 Vallo della Lucania, Salerno, Italy \\ 4 Dipartimento di Scienze dell'Ambiente, Università degli Studi di Napoli Parthenope, 80 I 43 Naples, Italy
}

Keywords: Anisakis simplex, Marinated anchovies, Colonoscopy

\section{SUMMARY}

Anisakidosis is a zoonotic disease caused by the ingestion of nematodes belonging to the family of Anisakidae. Human infection is caused by intake of raw or undercooked sea fish and cephalopods infested by Anisakis larvae. We present a case of accidental endoscopic finding of an alive nematode adhering to distal ascending colon in a 32 years old man, submitted to colonoscopy owing to recent onsets of rectal bleeding of likely hemorrhoidal origin. The nematode, removed from colon by means of biopsy forceps, has been identified as L3 larvae of $A$. simplex by a light microscope. Histological examination of intestinal mucosa showed a mild fibrosis of lamina propria, characterized by focal lymphocytic inflammation and scattered infiltration of eosinophils. The patient reported the intake of marinated anchovies 3 days before endoscopic examination.

\section{INTRODUCTION}

In 1960, Van Thiel, et al. (13) described in Holland for the first time an allergic reaction related to the presence of a marine nematode in a patient who manifested acute abdominal pain. The parasite causing the disease was subsequently identified as Anisakis spp. (14). Symptoms of human anisakidosis include violent abdominal pain, nausea, and vomiting or diarrhea. In some cases, Anisakis antigens produce allergic reactions and hypersensitivity (1). Acute or chronic diseases are described in gastric and intestinal anisakidiasis with a variety of histopathological patterns, which span from phlegmon formation to abscess or eosinophilic granuloma formation (12).

The life cycle of the nematode consists of several stages (egg $\rightarrow \mathrm{L} 1 \rightarrow \mathrm{L} 2 \rightarrow \mathrm{L} 3 \rightarrow \mathrm{L} 4 \rightarrow$ adult $)$ and the parasite, using big marine mammals (seals and dolphins) as definitive host, could infest human, as accidental host, after the ingestion of raw or uncooked seafood (sea fish or cephalopods) contaminated by larvae of the Anisakis (1).

Currently, seafood contaminated with Anisakis is very common: Kleter et al. (2009) (5), analysing the notifications reported through the European Commission's Rapid Alert System for Food and Feed (RASFF) over a four-year period (July 2003 - June 2007), observed that A. simplex was the second most predominant biological hazard. The notifications for Anisakis were exclusively associ- ated with sea-food. Salmon, herring, anchovies, scad, hake, and mackerel are among the most frequently parasitized fishes (3).

Since the ' $60 \mathrm{~s}$, because of the widespread consumption of raw fish in the population, many cases of anisakidosis have been reported in Japan (4); subsequently the disease caused by Anisakis was reported worldwide (2). Several clinical cases of patients infected by the parasite were also recorded in Italy following the ingestion of raw or undercooked fish contaminated with L3 larvae of Anisakis $(7,8)$. Recently the number of reported cases in Italy has increased since the first description in 1996 (11), however the real incidence of human anisakidosis is unknown; the disease seems to be more widespread in the regions of Southern and Central Italy (Abruzzo, Molise, Campania, Apulia, and Sicily) (6).

This paper deals with a case of accidental endoscopic finding of $A$. simplex in a 32 years old male patient come to our observation owing to recent onset of rectal bleeding of likely haemorrhoidal origin.

\section{Case}

A 32 years old male patient came to our observation for recent onset of episodes of rectal bleeding with no other symptomatology. A healthy systems and apparatus anamnesis was recorded with no evidence of any specific disease. Physical examination showed colonic normal sound without

\section{Corresponding author: Vincenzo Pasquale}

Dipartimento di Scienze dell'Ambiente, Università degli Studi di Napoli Parthenope,

Centro Direzionale, Isola C4, 80 I 43 Naples, Italy - Tel. +39 08I 5476529 - Fax +39 08I 54765I5

E-mail: vincenzo.pasquale@uniparthenope.it 
manifestation of systemic allergic reaction (urticaria, angioedema) or recent episodes of abdominal pain, vomiting, fever and diarrhea; the patient appeared in general good condition.

A retrospective clinical interview was performed and the patient referred that he had eaten raw anchovies, marinated in vinegar, three days before the colonoscopy

Colonoscopy. The total colonoscopy with retrograde ileoscopy, carried out in October 2010, showed at the distal ascending colon, near the hepatic flexure, a moderate salience hyperaemic and oedematous mucosa with profile slightly in relief. On the mucosal surface a small viable helminth was observed (Figure I). It was removed alive by biopsy forceps. No other injuries organic endoluminal were detected. In the anal canal moderately haemorrhoidal veins were also observed. Nevertheless, gastric and duodenum endoscopy was also performed to rule out the possibility of gastric form of anisakiasis and showed normal findings of body and antrum mucosa and minimal duodenum hyperaemia.

Laboratory analysis. The diagnosis of intestinal anisakiasis was based on light microscope morphologic characteristics of the helmint larva.

The parasite, fixed in $70 \%$ ethanol and cleared in glycerin, showing length and maximum-width $18 \times 0.4 \mathrm{~mm}$, prominent boring tooth, rounded tail with the presence of the mucron, and oblong ventriculus with oblique esophago-intestinal junction, was recognized as L3 larval stage of $A$. simplex (9). The following general hematological determinations were performed: leukocyte and eosinophil count, hemoglobin level, prothrombin time, partial thromboplastin time, reactive $\mathrm{C}$ protein, and erythrocyte sedimentation rate. All these parameters were in normal range. The results of the stool culture and parasites research were negative.

The immunology workup revealed serum immunoglobulin (IgM, IgA, $\operatorname{IgG}$, and total $\operatorname{IgE}$ ) levels to be within the normal range. Specific IgE to $A$. simplex, using an ImmunoCAP System (Phadia), was negative $(<0.35 \mathrm{KU} / \mathrm{L})$.

ImmunoCAP System is currently widely used in study of allergies in order to determine serum IgE antibodies against $A$. Simplex. According to the manufacturer's instructions, the test performed with undiluted serum allows the quantitative meausure of specific IgE antibodies in the range of 0.35 to $100 \mathrm{kU} / \mathrm{L}$.

The histological examination of multiple specimens revealed superficial inflammation, moderate mucosal oedema with soft fibrosis of lamina propria. In addition, we observed focal evidence of lymphocytic and eosinophilic cells (40-50 cells per HPF) without involvement of deeper layers of intestinal wall.

Treatment. The patient was discharged with albendazole ( $400 \mathrm{mg}$ b.i.d.) oral therapy for 4 days. He followed a diet without seafood ingestion and haematological analysis were repeated up to six weeks after infection and results were within the normal range.

\section{CONCLUSION}

The recurrent rectal bleeding, due moderately hemorrhoidal veins, requested an endoscopic analysis with accidental finding of a nematode identified as $A$. simplex (L3) at distal ascending colon of a 32 years old male patient.

Although the patient was asymptomatic, his ascending colon showed initial histological inflammation due to invasive infestation, after only 72 hours from anchovies ingestion. The reported case, the first in Salerno area (Southern Italy), confirms that human anisakidosis may occur, with no early intestinal or allergic symptom, following the ingestion of raw or undercooked fish, infested by Anisakis spp. larvae, which were not frozen at $-20^{\circ} \mathrm{C}$ for at least 24 hours after the catch, as required by Regulation (EC) $\mathrm{N}^{\circ} 853 / 2004$ (10).

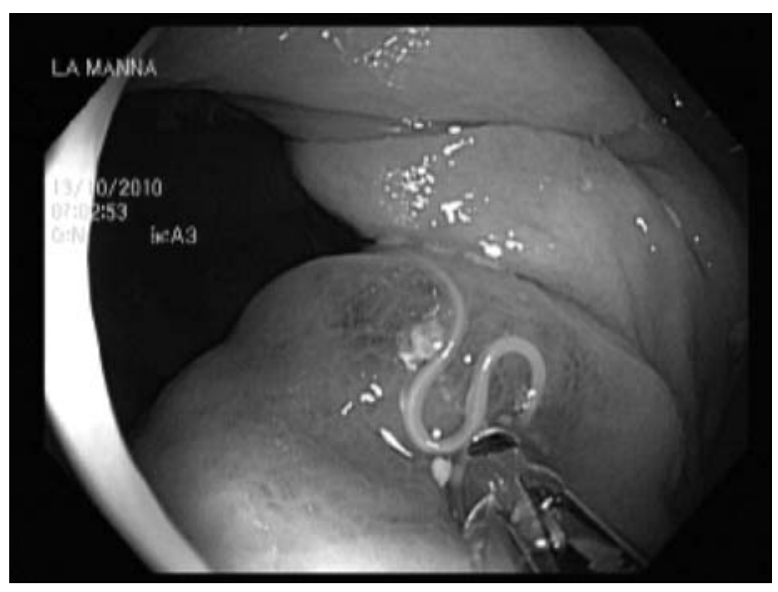

Figure I. Anisakis simplex adhering to hyperaemic and oedematous colic mucosa.

\section{REFERENCES}

1. Audicana MT, Kennedy MW. Anisakis simplex: from obscure infectious worm to inducer of immune hypersensitivity. Clin Microbiol Rev 2008; 21: 360-79.

2. Boure'e P, Paugam A, Petithory JC. Anisakidosis: report of 25 cases and review of the literature. Comp Immunol Microbiol Infect Dis 1995; 18: 75-84.

3. Hochberg NS, Hamer DH. Anisakidosis: Perils of the Deep. Emerg Infect 2010; 51: 806-12.

4. Ishikura H, Kikuchi K, Nagasawa K, et al. Anisakidae and anisakidosis, In: Progress in Clinical Parasitology, T. Sun (ed.). Springer-Verlag, New York, NY, 1993; 43-102.

5. Kleter GA, Prandini A, Filippi L, Marvin HJP. 
Identi?cation of potentially emerging food safety issues by analysis of reports published by the European Community's Rapid Alert System for Food and Feed (RASFF) during a four-year period. Food Chem Toxicol 2009; 47: 932-50.

6. Mattiucci S, Paoletti M, Borrini F, et al. First molecular identification of the zoonotic parasite Anisakis pegreffii (Nematoda: Anisakidae) in a paraffin-embedded granuloma taken from a case of human intestinal anisakiasis in Italy. BMC Infect Dis 2011; 11: 82.

7. Moschella CM, Mattiucci S, Mingazzini $P$, et al. Intestinal anisakiasis in Italy: case report. J Helminthol 2004; 78: 271-3.

8. Pampiglione S, Rivasi F, Criscuolo M, et al. Human anisakiasis in Italy: a report of eleven new cases. Pathol Res Pract 2002; 198: 429-34.
9. Pereira Bueno JM. Algunos aspectos de la epidemiologi'a y prevencio'n de la anisakidosis. Valladolid' Junta de Castilla y Leo' n. Consejeri'a de Sanidad y Bienestar Social; 1992.

10. Regulation (EC) $\mathrm{N}^{\circ} 853 / 2004$ of the Euopean Parliament and of the Council of 29 April 2004 laying down specific hygiene rules for food of animal origin.

11. Stallone O, Paggi L, Balestrazzi A, Mattiucci S, Montinari M. Gastric Anisakiasis in Italy: Case Report. Med J Sur Med 1996; 4: 13-6.

12. Takei H, Powell SZ. Intestinal anisakidosis (anisakiosis). Ann Diagn Pathol 2007; 11: 350-2.

13. Van Thiel PH, Kuipers FC, Roskam RT. A nematode parasitic to herring causing acute abdominal syndromes in man. Trop Geogr Med 1960; 12: 97-113.

14. Van Thiel PH. Anisakiasis. Parasitology 1962; 52: 16-7. 